\title{
Lower-limb joint kinetics in jump rope skills performed by competitive
}

athletes.

Olivia L. Bruce ${ }^{\mathrm{a}, \mathrm{b}}$, Mollee Ramsay ${ }^{\mathrm{a}}$, Geneva Kennedy ${ }^{\mathrm{a}}$, W. Brent

Edwards ${ }^{\mathrm{a}, \mathrm{b}}$

a. Human Performance Laboratory, Faculty of Kinesiology, University of Calgary,

Calgary, Canada $\quad$ b. Biomedical Engineering Graduate Program, University of

Calgary, Calgary, Canada

Corresponding Author: olivia.bruce@ucalgary.ca

"This is an Accepted Manuscript of an article published by Taylor \& Francis in Sports

Biomechanics on August 28, 2020, available at http://wwww.tandfonline.com/10.1080/14763141.2020.1801823." 


\section{Lower-limb joint kinetics in jump rope skills performed by competitive 2 athletes.}

3 Abstract

The purpose of this study was to characterise lower-limb joint kinetics during consecutive double unders and speed step sprints performed by competitive jump rope athletes, and to compare these measurements to running. Sixteen adolescent competitive jump rope athletes performed consecutive double under, speed step, and running trials while motion capture and ground reaction force data were collected. Lower limb joint moments, power, and work were calculated using an inverse dynamics approach and discrete measurements were compared between skills. Peak ground reaction forces were similar between movements; however, knee and hip joint kinetics were distributed differently between double unders and speed step. In general, double unders were characterised by an increased reliance on knee joint kinetics, while speed step was characterised by an increased reliance on hip joint kinetics. Peak ankle moments were 9-20\% greater in speed step when compared to double unders and running ( $\mathrm{p} \leq 0.050)$, and peak negative ankle power was $39-114 \%$ greater in double unders and speed step when compared to running $(\mathrm{p} \leq 0.002)$. These findings may have important implications for injury risk and load management in jump rope athletes or other individuals that incorporate jump rope into their training programs.

Keywords: biomechanics; musculoskeletal system; lower extremity; rope skipping; hopping

Introduction: Jump rope is an activity widely used in physical education, fitness, and sport training programs for its cardiorespiratory (Quirk \& Sinning, 1982), strength (Duzgun et al., 2010), agility (Miyaguchi et al., 2015), coordination (Ozer et al., 2011), and bone health (Pettersson et al., 2000) benefits. One million students across 4,000 schools in Canada participate in Jump Rope for Heart programs annually (Heart and Stroke Foundation of Canada, n.d.). In addition to its use in fitness and recreation, jump rope is a growing competitive sport with clubs in 51 countries (International Jump Rope 
Union, n.d.). Athletes compete individually and as teams in events including timed speed, consecutive triple unders, and freestyle (International Jump Rope Union, n.d.). While there is a wide variety of skills performed by these athletes, jump rope may be characterised by highly repetitive hopping with closely coupled upper limb movements controlling rope turning.

It has been hypothesised that the development of overuse injuries, though multifactorial and variable between specific injuries, is associated with repetitive loading and insufficient recovery time (Edwards, 2018). Repetitive loading, especially at high magnitudes, results in microdamage accumulation in both tendon and bone (Pattin et al., 1996; Wren et al., 2003). Without adequate repair, the accumulation of damage may eventually lead to injury (e.g., tendinopathy or stress fracture) (Edwards, 2018). in competitive jump rope (Ollivierre, 1994); these injuries are also prevalent in other jumping/running sports such as volleyball and basketball (Eerkes, 2012; Gordon et al., 2014).

Previous jump rope studies have reported ground reaction force (GRF) (Jang et al., 2017; Kim et al., 2017; Pittenger et al., 2002; Shek et al., 2005), plantar pressure (Shek et al., 2005), and joint angle (Jang et al., 2017; Kim et al., 2017) parameters. Most of the studies quantified external loading parameters or kinematics for one- or two-legged single unders (one revolution of the rope per jump) or double unders (two revolutions per jump; see Online Supplementary video 1). Both movements involve continuous hopping, where the lower limbs are relatively rigid and the majority of jumping forces are produced by the ankle and knee (Lamontagne \& Kennedy, 2013). Shek et al. (2005) also quantified GRF and plantar pressure for speed step (see Online Supplementary video 2) and consecutive triple unders (three revolutions per jump; see Online Supplementary video 
3), performed by one elite jump rope athlete. Consecutive triple unders require greater jump height and rope speed compared to double unders and are competed among athletes age 15 years and older. Speed step, also referred to as 'alternating step,' is primarily used for timed speed events. Unlike single and multiple unders, speed step involves jumping from one leg to the other using a stepping motion that enables the athlete to achieve a rapid pace. Shorter ground contact time has also been observed during speed step compared to single or multiple unders (Shek et al., 2005).

To date, jump rope biomechanics studies have focused on GRF and kinematics. Only two of these studies have investigated multiple unders or speed step (Kim et al., 2017; Shek et al., 2005), which are fundamental, repetitive skills that make up a large proportion of a competitive athlete's training. No study has reported joint kinetics, which are indicative of the magnitude and distribution of musculoskeletal loading. Thus, the purpose of this study was to characterise joint kinetics during consecutive double unders and speed step sprints performed by competitive jump rope athletes. Parameters were also compared to running, which is a widely studied repetitive loading activity with a high incidence of lower-limb overuse injuries (Fukuchi et al., 2017; Messier et al., 2018; Schache et al., 2011; Scott \& Winter, 1990; Tenforde et al., 2011). Differences exist between double unders and speed step with respect to kinematics, jump height, and ground contact time (Shek et al., 2005), which are likely to influence joint kinetic parameters. For example, a previous study observed differences in the distribution of joint moments throughout the lower limb associated with different ground contact times during hopping (Hobara et al., 2010). Therefore, we hypothesised that the distribution and magnitude of lower limb joint kinetic parameters would be different between double unders and speed step. 
Methods: Sixteen adolescent competitive jump rope athletes (14 F and $2 \mathrm{M}, 14.3 \pm 1.8$ years, $1.63 \pm 0.08 \mathrm{~m}, 59.7 \pm 8.1 \mathrm{~kg}$ ) were recruited from teams in Alberta, Canada. Athletes competed at the provincial level or above in the previous season and had no musculoskeletal injuries in the three months prior to testing that would keep them from training. All testing procedures were approved by the university's Conjoint Health Research Ethics Board. All participants, and guardians for participants aged 15 or younger, provided written informed consent.

Each athlete performed their typical warm-up, which generally consisted of a series of dynamic stretches and light bodyweight exercises targeting the lower and upper limbs, and core. Retroreflective markers were then placed at landmarks on the pelvis, dominant leg, and shoe (i.e., sacrum, right and left anterior superior iliac spine and greater trochanters, proximal anterior thigh, distal anterior thigh, lateral thigh, lateral and medial knee, proximal anterior shank, distal anterior shank, posterior shank, lateral and medial malleoli, dorsal foot, first metatarsal head, third metatarsal head, fifth metatarsal head, distal hallux, and calcaneus) (Bruce et al., 2019). Following a static motion-capture trial, participants performed running, consecutive double unders, and speed step sprint trials while motion capture $(240 \mathrm{~Hz}, 8$ high-speed Eagle cameras; Motion Analysis Corporation, Santa Rosa, CA, USA) and force platform (1 force plate, $2400 \mathrm{~Hz}$, Kistler Instrument Corp., Amherst, NY, USA) data were collected synchronously using Cortex software (v5.0, Motion Analysis Corporation, Santa Rosa, CA, USA). Participant anthropometrics (height, mass, lower limb segment lengths and circumferences) were 100 recorded at the end of the session. Running trials were performed at $3.5 \mathrm{~m} / \mathrm{s} \pm 5 \%$ along a $15 \mathrm{~m}$ lane. This speed was chosen because it is commonly used in running studies, enabling comparison to previous literature (Fukuchi et al., 2017; Schache et al., 2011). Participants completed practice runs 
104 prior to data collection until they were able to achieve the target speed consistently. Three successful trials were collected. Trials were repeated if the dominant leg did not land on the force platform, the participant did not achieve the target speed, or if the participant appeared to change their cadence in order to target the force platform.

For the jump rope movements, athletes used their own custom wire ropes (R1

109 Speed, JumpNrope, Ltd., CO, USA, or Elite Surge, BuyJumpRopes.net, WA, USA, with

110 coated or uncoated stainless steel cords) for testing. Athletes performed single and double

111 unders to practice prior to the trial. Each athlete then performed 20-30 consecutive double

112 unders (Online Supplementary video 1) at self-selected jump frequency with their

113 dominant foot on the force platform and non-dominant foot on the ground adjacent. If the

114 athlete drifted such that the markered foot landed partially off the force platform or the

115 non-markered foot landed on the force platform, verbal instructions were provided until

116 that athlete was correctly aligned. Frontal plane video of the feet and force platform was

117 recorded for the purpose of identifying incorrectly aligned landings to be excluded from 118 data analysis.

119 Athletes then performed the speed step trial. A practice trial was completed at 120 approximately $70 \%$ of maximum speed. Following a minimum one minute rest, each 121 athlete performed a maximal $10 \mathrm{~s}$ sprint using the speed step (Online Supplementary video 2). The trial was repeated if more than one miss (where the rope stopped due to catching on the body) occurred. Three of the participants $(2 \mathrm{~F}, 1 \mathrm{M}, 15-17$ years, $1.57-1.67 \mathrm{~m}, 56.9-61.2 \mathrm{~kg})$ were 125 also able to complete a trial of 15-25 consecutive triple unders (Online Supplementary video 3). An opportunistic analysis was performed using the same protocol as double unders. 
129 GRF data were down-sampled to match motion capture data. Data were then cut to isolate

130 the ground contact phases. For running trials, heel strike was defined as the first data point

$131 \geq 20 \mathrm{~N}$. Toe-off was defined as the first data point following heel strike $\leq 20 \mathrm{~N}$. For the

132 consecutive double unders and triple unders, the ground contact phase following the first

133 double/triple under was excluded, as the first jump is usually higher to accommodate the

134 initial slower rope velocity. Contact phases where the markered foot was partially off the

135 force platform, or where the non-markered foot was on the force platform (identified

136 through a conservative visual inspection of the video recorded during the trial) were

137 excluded. The first 10 contact phases of the remaining double unders, and the first 8

138 contact phases of the remaining triple unders, were selected for analysis. Touch-down

139 and toe-off were defined using a $20 \mathrm{~N}$ threshold similar to the running trials. For the speed

140 step trial, the first two contact phases of the markered leg were excluded, as the athlete

141 increases speed over the initial steps. The next 10 contact phases corresponding to the

142 markered leg were selected for analysis. Again, touch-down and toe-off were defined

143 using a $20 \mathrm{~N}$ threshold.

144 Retro-reflective markers were used to define local reference frames for the pelvis,

145 thigh, shank, and foot. Cardan segment and joint angles were calculated using a flexion-

146 extension, abduction-adduction, internal-external rotation sequence. Segment masses,

147 centre of mass locations, and moments of inertia for the thigh, shank, and foot were

148 obtained using anthropometric measures and equations described by Vaughan et al.

149 (1999). An inverse dynamics approach was used to calculate joint moments in the global

150 reference frame and these were subsequently transformed into the local reference frame

151 of the distal segment. Joint power was calculated by multiplying the joint moment by the

152 joint angular velocity. Segment angular accelerations for inverse dynamics analysis and 
153 joint angular velocities for joint power calculations were obtained using the finite

154 difference method. A $4^{\text {th }}$ order zero-lag Butterworth filter was used to smooth the raw

155 GRF, joint moment, and power data at the 95th percentile frequency of the distal vertical

156 reaction force (Edwards, Troy, \& Derrick, 2011). Vertical instantaneous loading rate

157 (VILR) was calculated as the slope of the vertical GRF using the finite difference method.

158 Impulse was calculated as the time-integral of the vertical GRF. Positive and negative

159 joint work were calculated as the time-integral of positive and negative joint power,

160 respectively.

161 Cycle-averaged peak vertical GRF, impulse, peak vertical instantaneous loading

162 rate (VILR), peak net joint moments, peak positive and negative joint powers, and

163 positive and negative joint work were quantified for each athlete. Analysis of joint

164 kinetics was limited to those occurring about the segment medial-lateral (i.e., flexion-

165 extension) axis, the predominant axis of motion for double unders, speed step, and

166 running. Each variable was checked for normality using the Shapiro-Wilk test and visual

167 examination of histograms and Q-Q plots. Repeated measures ANOVAs were used to test

168 for differences among the movements. Where relevant, post-hoc paired t-tests with a

169 Bonferroni correction were used to determine differences between pairs of running,

170 double unders, and speed step. If data were non-normal, a log transform was applied and

171 normality was checked again. If the data were still not normally distributed, a Friedman

172 test was used to test for differences between movements. Wilcoxon signed-rank tests were

173 used for post-hoc analysis where relevant. Effect sizes (partial $\eta^{2}$ for ANOVAs and

174 average Spearman rho for Friedman tests) were calculated for main effects. All statistical

175 tests were performed in SPSS (v.24.1.1, IBM, Armonk, NY, USA) with a criterion alpha-

176 level of 0.05 . 
177 Results: The average cadence for double unders and speed step was $2.0 \pm 0.2$ and $4.9 \pm$

1780.4 jumps/s, respectively. Significant main effects were observed for stance time,

179 impulse, and VILR $\left(\mathrm{p} \leq 0.003, \eta^{2} \geq 0.379\right.$, Table 1$)$. Stance time was $27 \%$ and $41 \%$

180 shorter (95\% CI: $0.051-0.085 \mathrm{~s}$ and $0.080-0.120 \mathrm{~s}$ ), and vertical impulse was $30 \%$ and

$18143 \%$ lower (CI: $51.2-81.7$ and $70.8-112.7 \mathrm{BW} \cdot \mathrm{s})$, for double unders and speed step,

182 respectively, when compared to running ( $\mathrm{p} \leq 0.001)$. Peak VILR was $47 \%$ greater

183 during speed step when compared to double unders ( $\mathrm{p}<0.001$, CI (ratio of means): 1.26

$184-1.72)$. No differences in peak vertical GRF were observed $(p=0.240)$.

185 Significant main effects were observed for all joint kinetic parameters $(\mathrm{p} \leq 0.023$,

$186 \eta^{2} \geq 0.223$ ) except peak positive ankle power, peak negative knee power, and negative

187 ankle work (Table 1). Peak ankle moment and negative power were greater during speed

188 step than running and double unders $\left(\mathrm{p} \leq 0.050, \mathrm{CI}_{\text {moment: }} 0.02-29.9 \mathrm{Nm}\right.$ and $15.3-46.0$

$189 \mathrm{Nm}, \mathrm{CI}_{\text {power }}$ (ratio): $1.45-3.16$ and $1.17-2.03$ ). Negative ankle power was also greater

190 during double unders when compared to running ( $\mathrm{p}=0.002$, CI (ratio): $1.14-1.70$ ).

191 Positive ankle work was lower during speed step than double unders and running ( $\mathrm{p} \leq$

192 0.001, CI: $4.5-14.3 \mathrm{~J}$ and $7.3-17.5 \mathrm{~J}$ ). Peak knee moment (Figure 1) and positive work

193 (Figure 2) were lower in speed step than running and double unders $\left(\mathrm{p} \leq 0.004, \mathrm{CI}_{\text {moment: }}\right.$ :

$19427.9-77.1 \mathrm{Nm}$ and $50.5-105.0 \mathrm{Nm}$ ). Negative knee work was also lower in speed step

195 than running $(\mathrm{p}=0.008)$ and peak knee moment was greater in double unders when

196 compared to running $\left(\mathrm{p}=0.036, \mathrm{CI}_{\text {moment }}: 1.4-49.1 \mathrm{Nm}\right)$. All hip joint kinetic parameters

197 were lower in double unders when compared to running and speed step $(\mathrm{p} \leq 0.023$, Table

198 1, Figures 1 and 2). Hip positive work was lower, and peak powers were greater, in speed

199 step when compared to running ( $\mathrm{p} \leq 0.034, \mathrm{CI}_{\mathrm{work}}$ : $6.9-20.3 \mathrm{~J}, \mathrm{CI}_{\text {power, positive }}$ (ratio): 1.21

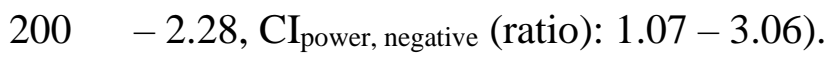


While stance time was similar to double unders, all other measures (peak vertical

202 GRF, impulse, VILR, peak joint moments, peak joint powers, and joint work) tended to

203 be larger in triple unders when compared to double unders (Table 2, Figure 3).

204 Discussion and implications: The purpose of this study was to characterise joint

205 kinetics during consecutive double unders and speed step in competitive jump rope

206 athletes, and to compare these measurements to running. Although peak GRF was

207 similar between movements, knee and hip joint kinetics were distributed differently

208 between double unders and speed step. In general, double unders were characterised by

209 an increased reliance on knee joint kinetics, while speed step was characterised by an

210 increased reliance on hip joint kinetics. Peak ankle moments were 9-20\% greater in

211 speed step when compared to double unders and running $(\mathrm{p} \leq 0.050)$, and peak negative

212 ankle power was 39-114\% greater in double unders and speed step when compared to

213 running $(\mathrm{p} \leq 0.002)$. These findings may have important implications for injury risk and

214 load management in jump rope athletes or other individuals that incorporate jump rope

215 into their training programs.

216 Across all movements, the greatest joint kinetic parameters were observed at the

217 ankle. Peak ankle moments were $20 \%$ and $9 \%$ greater in speed step than double unders

218 and running, respectively, and peak negative ankle power was $39-114 \%$ greater in the

219 jump rope skills when compared to running. These larger loads about the ankle joint,

220 produced primarily by the triceps surae muscles, are transmitted to the calcaneus via the

221 Achilles tendon (Fukashiro et al., 1995; Lamontagne \& Kennedy, 2013). Greater forces

222 through the Achilles tendon would also result in greater ankle joint contact force and

223 bending moments applied to the tibia (Scott \& Winter, 1990). For musculoskeletal tissues,

224 such as tendon and bone, it is well known that fatigue life measurements are heavily

225 influenced by loading magnitude (Pattin et al., 1996; Wren et al., 2003), but it is also 
important that the number of loading cycles is considered (Edwards, 2018). Speed step and double unders are usually performed in a series of short bouts (10 s to $5 \mathrm{~min}$ ). In a typical training session, an athlete may perform a cumulative total of 20 minutes of speed step at an average of $90 \%$ of their maximum speed, resulting in 2,646 steps per foot. Adding cycles from consecutive double unders, typically 500 per session, would result in a total of 3,146 cycles per foot. For comparison, a 30-minute run with a cadence of 85 strides/minute (Fukuchi et al., 2017) would result in 2,550 strides. It is well known that runners have a high incidence of overuse injuries, such as Achilles tendinopathy and tibial stress fractures (Tenforde et al., 2011). Given the similar or greater magnitudes of musculoskeletal loading and the high number of cycles associated with jump rope speed/double unders training sessions, a high incidence of overuse injuries at the ankle and lower limb would be expected in jump rope athletes. Indeed, these types of injuries have been documented (Ollivierre, 1994).

Although peak GRF was not different between movements, differences in joint kinetic patterns were observed at the knee and hip. Peak knee extension moment was

241 minimal in speed step, being $61 \%$ lower when compared to running. The lower knee 242 moment could be explained, in part, by the shorter ground contact time as a previous study of vertical hopping observed a decrease in peak knee moment with increasing hop

244 frequency (and associated decrease in ground contact time) (Hobara et al., 2010). Leg 245 stiffness was also previously observed to increase with hop frequency, and this was 246 achieved primarily through changes at the knee and hip (Hobara et al., 2010). The 247 stepping motion used in speed step explains the distinct patterns in knee and hip joint power (Figure 2). Unlike double unders and running, knee and hip extension occurs in

249 the first half of ground contact, followed by flexion in the second half (Figure 4). The 250 positive power at the knee and hip in the first half of ground contact indicates concentric 
muscle contraction extending the joints. The negative power at the knee, corresponding

252 with a flexion moment, in the first half of ground contact during speed step indicates eccentric muscle activity slowing down extension of the knee. The active extension of the leg in the first half of ground contact, combined with the shorter contact time, may explain the greater VILR compared to double unders, and the larger peak negative power at the ankle compared to double unders and running. In the second half of ground contact, negative power and moment, and positive angular velocity were observed at the hip. Taken together, this indicates eccentric muscle activity acting to resist hip flexion. Both knee and hip flexion were observed throughout the second half of ground contact during speed step, in contrast with extension at both joints during double unders and running (Figure 4). This suggest the majority of propulsive force during speed step is generated at the ankle.

In double unders, the kinetic parameters were highest at the ankle and knee, and this distribution of joint loading is consistent with submaximal vertical hopping (Souza et al., 2010). Compared to running, peak knee extension moment and positive power were $29 \%$ and $69 \%$ greater, respectively, and all hip joint kinetic parameters were $36-84 \%$ lower. Similarly, peak knee moment and positive work were $233 \%$ and $179 \%$ greater in double unders when compared to speed step. Joint kinetic parameters are indicative of the magnitude of musculoskeletal loads. It may be important to consider the different distribution of joint kinetics at the ankle, knee, and hip between double unders and speed

271 step when creating training programs for jump rope athletes or when choosing which 272 jump rope skill to incorporate into cross training programs. For example, an athlete specialising in multiple unders and freestyle events may consider incorporating additional exercises to improve knee extension strength into their off-season training. Athletes in sports other than jump rope often use double unders in cross training workouts to increase 
cardiovascular demand. Speed step could be used as an alternative in a workout that involves other exercises which impose a heavy load on the knee.

unders, instead of double unders. Kinetic parameters across all joints tended to be greater during triple unders when compared to double unders (Table 2, Figure 3). Similar to double unders, athletes typically perform fewer triple unders (100-500) per session compared to speed step or running training. Due to the ages of participants in the current study, only three athletes were able to complete 15-25 consecutive triple unders. Given the greater magnitudes of the joint kinetic parameters, further work evaluating the training load associated with triple unders performed by older athletes is warranted.

A limitation of the present study is the large range of ages, statures, and skill levels (speed scores and competitive experience) present in the participant group tested. It is possible that technique differences may exist between athletes of different ages or skill levels (Jang et al., 2017; Kim et al., 2017). While the sprint speeds observed in this study $(4.9 \mathrm{~Hz})$ are representative of typical scores for this age group at the provincial level in Alberta, greater speeds $(7.5 \mathrm{~Hz})$ have been observed at the international level

292 (International Jump Rope Union, n.d.). Further work is needed to determine how speed influences joint kinetics during speed step. The broad inclusion criteria were necessary due to the limited number of jump rope athletes near the university. While the competitive jump rope population is small relative to sports such as running, these results are relevant to other populations. For example, Cross-Fit athletes perform double unders in training 297 and competition, often in combination with running and other exercises.

298 Conclusion: Joint kinetics in competitive athletes performing jump rope skills were on 299 the order of magnitude, and in some instances higher, than joint kinetics in running. The 300 high joint kinetic magnitudes, especially at the ankle, combined with high repetition 
301 may suggest these athletes and others incorporating jump rope into training may be at

302 risk of developing overuse injuries. Differences in joint kinetic distributions at the knee

303 and hip between jump rope skills should also be considered when structuring training

304 programs.

305

306 Acknowledgements

307 The authors would like to acknowledge Calgary Skip Time, Calgary Jump Crew,

308 and Connectivity Skippers for their assistance with recruitment and the participation of

309 some of their athletes in this study.

310

311 Disclosure of Interest: This research did not receive any specific grant from funding

312 agencies in the public, commercial, or not-for-profit sectors.

313 
315 Bruce, O. L., Firminger, C. R., Wannop, J. W., Stefanyshyn, D. J., \& Edwards, W. B. (2019). Effects of basketball court construction and shoe stiffness on countermovement jump landings. Footwear Science, 11(3), 171-179. https://doi.org/10.1080/19424280.2019.1668867

Duzgun, I., Baltaci, G., Colakoglu, F., Tunay, V. B., \& Ozer, D. (2010). The effects of jump-rope training on shoulder isokinetic strength in adolescent volleyball players. Journal of Sport Rehabilitation, 19(2), 184-199.

Edwards, W. B. (2018). Modeling Overuse Injuries in Sport as a Mechanical Fatigue Phenomenon. Exercise and Sport Sciences Reviews, 46(4), 224-231.

Edwards, W. B., Troy, K. L., \& Derrick, T. R. (2011). On the filtering of intersegmental loads during running. Gait and Posture, 34(3), 435-438.

Eerkes, K. (2012). Volleyball injuries. Current Sports Medicine Reports, 11(5), 251https://doi.org/10.1016/j.gaitpost.2011.06.006

330 Fukashiro, S., Komi, P. V, Jarvinen, M., \& Miyashita, M. (1995). In vivo Achilles tendon loading during jumping in humans. European Journal of Applied Physiology and Occupational Physiology, 71(5), 453-458.

334 Fukuchi, R. K., Fukuchi, C. A., \& Duarte, M. (2017). A public dataset of running biomechanics and the effects of running speed on lower extremity kinematics and kinetics. PeerJ, 5, e3298. https://doi.org/10.7717/peerj.3298

337 Gordon, A. I., Di Stefano, L. J., Denegar, C. R., Ragle, R. B., \& Norman, J. R. (2014). 338 College and professional women's basketball players' lower extremity injuries: A 
survey of career incidence. International Journal of Athletic Therapy and Training, 19(5), 25-33. https://doi.org/10.1123/ijatt.2014-0020

Heart and Stroke Foundation of Canada. (n.d.). Jump Rope for Heart. https://securesupport.heartandstroke.ca/site/SPageServer/?pagename=jump_home\&s_locale=en - CA

Hobara, H., Inoue, K., Muraoka, T., Omuro, K., Sakamoto, M., \& Kanosue, K. (2010). Leg stiffness adjustment for a range of hopping frequencies in humans. Journal of Biomechanics, 43(3), 506-511. https://doi.org/10.1016/j.jbiomech.2009.09.040

International Jump Rope Union. (n.d.). IJRU. https://ijru.sport/ (2017). Effects of Skill Level and Feet Width on Kinematic and Kinetic Variables during Jump Rope Single Under. Korean Journal of Sport Biomechanics, 27(2), 99-108. https://doi.org/10.5103/kjsb.2017.27.2.99

Kim, D. Y., Jang, K. H., Lee, M. G., Son, M. J., Kim, Y. K., Kim, J. H., \& Youm, C. H. (2017). Analysis of Kinematics and Kinetics According to Skill Level and Sex in Double-under Jump Rope Technique. Korean Journal of Sport Biomechanics, 27(3), 171-179. https://doi.org/10.5103/kjsb.2017.27.3.171

Lamontagne, M., \& Kennedy, M. J. (2013). The biomechanics of vertical hopping: a review. Research in Sports Medicine, 21(4), 380-394. https://doi.org/10.1080/15438627.2013.825795

Messier, S., Martin, D., Mihalko, S., Ip, E., Devita, P., Cannon, D., Love, M., Beringer, D., Saldana, S., Fellin, R., Seay, J. (2018). A 2-Year Prospective Cohort Study of Overuse Running Injuries: The Runners and Injury Longitudinal Study (TRAILS). The American Journal of Sports Medicine, 46(9), 2211-2221. https://doi.org/10.1177/0363546518773755 
364 Miyaguchi, K., Demura, S., \& Omoya, M. (2015). Relationship Between Jump Rope

365 Double Unders and Sprint Performance in Elementary Schoolchildren. Journal of

$366 \quad$ Strength and Conditioning Research, 29(11), 3229-3233.

367 https://doi.org/10.1519/JSC.0000000000000543

368 Ollivierre, R. (1994). Frequency and etiology of rope skipping injuries in children

369 participating in the International Rope Skipping Organization [Master's Thesis,

370 Temple University]. ProQuest Dissertations and Theses.

371 Ozer, D., Duzgun, I., Baltaci, G., Karacan, S., \& Colakoglu, F. (2011). The effects of

372 rope or weighted rope jump training on strength, coordination and proprioception

373 in adolescent female volleyball players. The Journal of Sports Medicine and

$374 \quad$ Physical Fitness, 51(2), 211-219.

375 Pattin, C. A., Caler, W. E., \& Carter, D. R. (1996). Cyclic mechanical property

376 degradation during fatigue loading of cortical bone. Journal of Biomechanics, 29(1), 69-79. https://doi.org/10.1016/0021-9290(94)00156-1

378 Pettersson, U., Nordstro, P., \& Alfredson, H. (2000). Effect of High Impact Activity on 379 Bone Mass and Size in Adolescent Females : A Comparative Study Between Two 380 Different Types of Sports. Calcified Tissue International, 67, 207-214.

$381 \quad$ https://doi.org/10.1007/s002230001131

382 Pittenger, V. M., McCaw, S. T., \& Thomas, D. O. (2002). Vertical ground reaction 383 forces of children during one- and two-leg rope jumping. Research Quarterly for $384 \quad$ Exercise and Sport, 73(4), 445-449.

$385 \quad$ https://doi.org/10.1080/02701367.2002.10609044

386 Quirk, J. E., \& Sinning, W. E. (1982). Anaerobic and aerobic responses of males and 387 females to rope skipping. Medicine and Science in Sports and Exercise, 14(1), 26388 29. 
Schache, A. G., Blanch, P. D., Dorn, T. W., Brown, N. A. T., Rosemond, D., \& Pandy, M. G. (2011). Effect of running speed on lower limb joint kinetics. Medicine and Science in Sports and Exercise, 43(7), 1260-1271. https://doi.org/10.1249/MSS.0b013e3182084929

Scott, S., \& Winter, D. A. (1990). Internal forces of chronic running injury sites. Medicine and Science in Sports and Exercise, 22(3), 357-369. https://doi.org/10.1249/00005768-199006000-00013

Shek, M. C., Fong, D. T. P., \& Hong, Y. (2005). Ground reaction forces and plantar kinetics of rope skipping in different sports shoes - A pilot study. In 23 International Symposium on Biomechanics in Sports (pp. 238-241). Beijing, China.

Souza, R. B., Arya, S., Pollard, C. D., Salem, G., \& Kulig, K. (2010). Patellar tendinopathy alters the distribution of lower extremity net joint moments during hopping. Journal of Applied Biomechanics, 26(3), 249-255. https://doi.org/10.1123/jab.26.3.249

Tenforde, A. S., Sayres, L. C., McCurdy, M. L., Collado, H., Sainani, K. L., \& Fredericson, M. (2011). Overuse Injuries in High School Runners: Lifetime Prevalence and Prevention Strategies. PM and R, 3(2), 125-131. https://doi.org/10.1016/j.pmrj.2010.09.009 ed). Kiboho Publishers. and cyclic loading on the mechanical properties and failure of human Achilles tendons. Annals of Biomedical Engineering, 31, 710-717. 


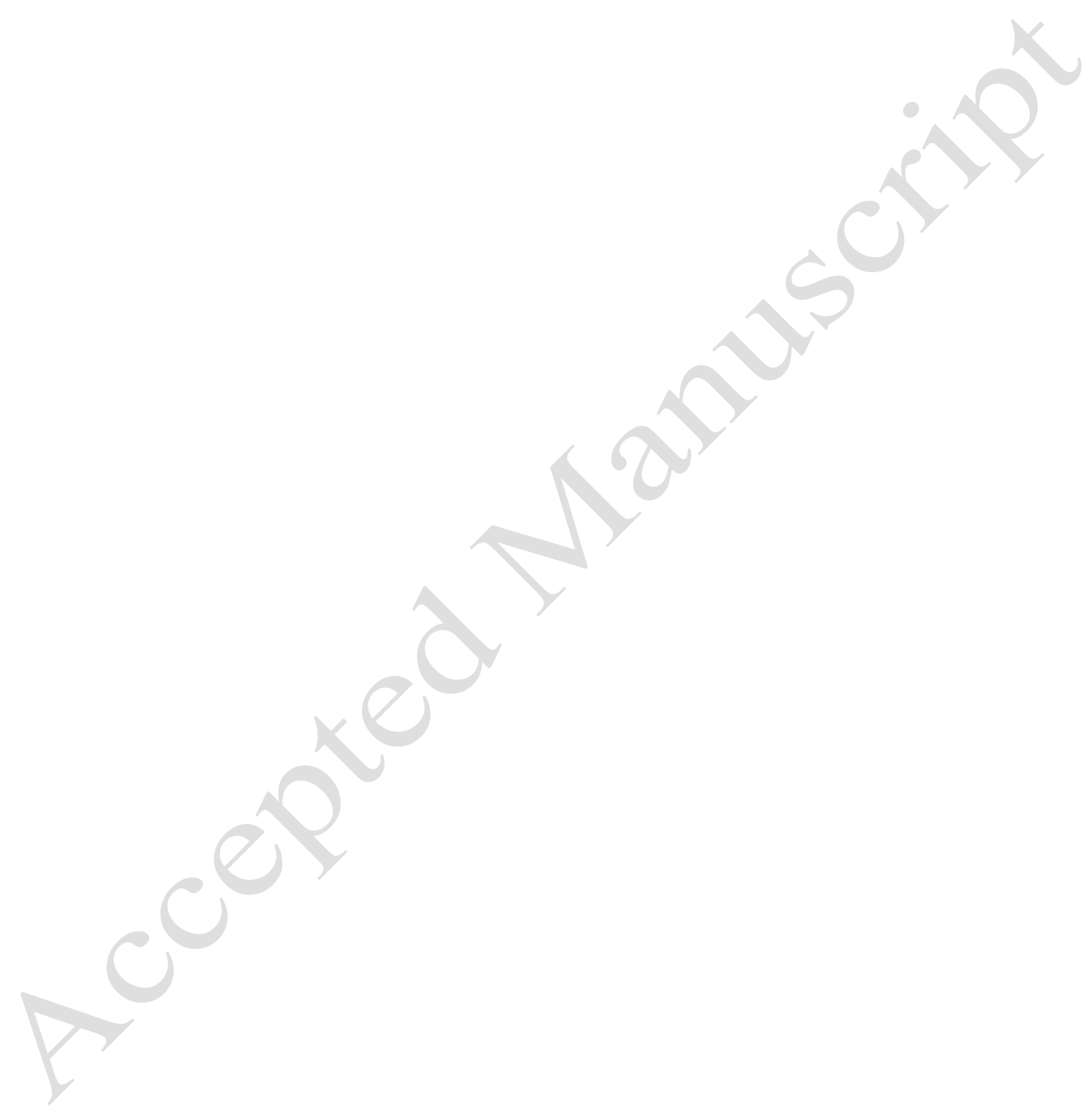


415 Table 1. Mean (95\% CI) ground reaction force and joint kinetic parameters at the ankle, 416 knee, and hip for running, consecutive double unders, and speed step.

\begin{tabular}{|c|c|c|c|c|c|}
\hline Measure & Running & Double Unders & Speed Step & p-value & $\begin{array}{l}\text { Effec } \\
\text { t size }\end{array}$ \\
\hline Contact time (s) & $0.245(0.232-0.258)$ & $0.178(0.165-0.190) \mathrm{a}$ & $0.145(0.134-0.156) a, b$ & $<0.001$ & 0.892 \\
\hline vGRF max (BW) & $2.54(2.45-2.63)$ & $2.62(2.44-2.79)$ & $2.66(2.51-2.82)$ & 0.240 & - \\
\hline Impulse $(\mathrm{BW} \cdot \mathrm{s})$ & $214.9(192.2-237.7)$ & $148.5(132.7-164.2) \mathrm{a}$ & $123.1(110.7-135.6) a, b$ & $<0.001$ & 0.882 \\
\hline VILR max $(\mathrm{BW} / \mathrm{s})^{*}$ & $63.2(54.2-73.6)$ & $48.9(43.1-55.5)$ & $71.8(62.2-83.0) \mathrm{b}$ & 0.003 & 0.379 \\
\hline \multicolumn{6}{|l|}{ Peak joint moment $(\mathrm{Nm})$} \\
\hline $\begin{array}{l}\text { ankle } \\
\text { plantarflexion }\end{array}$ & $169.2(149.9-188.4)$ & $153.5(136.3-170.7)$ & $184.1(163.8-204.5) a, b$ & $<0.001$ & 0.480 \\
\hline knee extension & $85.9(68.9-103.0)$ & $111.2(88.4-134.0) \mathrm{a}$ & $33.4(19.2-47.7) a, b$ & $<0.001$ & 0.704 \\
\hline hip extension & $140.5(123.5-157.4)$ & $68.5(57.0-80.0) \mathrm{a}$ & $121.0(106.0-136.0) b$ & $<0.001$ & 0.704 \\
\hline \multicolumn{6}{|l|}{$\begin{array}{l}\text { Peak positive joint power } \\
\text { (W) }\end{array}$} \\
\hline ankle** & $614.9(212.9)$ & $746.1(230.9)$ & $650.1(220.0)$ & 0.099 & - \\
\hline knee* & $192.3(155.2-238.2)$ & $324.3(236.0-445.7) \mathrm{a}$ & $216.8(141.3-178.6)$ & 0.023 & 0.223 \\
\hline hip* & $396.3(329.6-476.4)$ & $116.9(80.0-170.0) \mathrm{a}$ & $657.7(526.0-824.1) \mathrm{a}, \mathrm{b}$ & $<0.001$ & 0.828 \\
\hline \multicolumn{6}{|l|}{$\begin{array}{l}\text { Peak negative joint power } \\
\text { (W) }\end{array}$} \\
\hline ankle* & $544.5(448.7-662.2)$ & $756.8(650.1-881.0) \mathrm{a}$ & $\begin{array}{l}1166.8(922.6- \\
1472.3) a, b\end{array}$ & $<0.001$ & 0.559 \\
\hline knee* & $385.5(267.9-554.6)$ & $262.4(185.4-371.5)$ & $332.7(232.8-476.4)$ & 0.251 & - \\
\hline hip* & $191.4(140.9-260.0)$ & $122.2(95.5-156.3) \mathrm{a}$ & 345.9 (271.6-441.6) a,b & $<0.001$ & 0.574 \\
\hline \multicolumn{6}{|l|}{ Positive work (J) } \\
\hline ankle & $40.2(34.8-45.6)$ & $37.2(32.3-42.2)$ & $27.8(24.2-31.4) \mathrm{a}, \mathrm{b}$ & $<0.001$ & 0.646 \\
\hline knee** & $12.4(8.8)$ & $15.6(13.3)$ & $5.6(5.6) \mathrm{a}, \mathrm{b}$ & 0.002 & 0.362 \\
\hline hip & $35.7(28.9-42.5)$ & $5.7(3.8-7.5) \mathrm{a}$ & $22.1(19.0-25.2) a, b$ & $<0.001$ & 0.840 \\
\hline \multicolumn{6}{|l|}{ Negative work (J) } \\
\hline ankle & $41.0(39.1-50.1)$ & $38.5(32.7-44.3)$ & $33.1(28.4-37.8)$ & 0.167 & - \\
\hline knee** & $13.7(17.6)$ & $11.2(15.0)$ & $7.4(4.2) \mathrm{a}$ & 0.018 & 0.200 \\
\hline hip** & $5.5(5.0)$ & $2.7(3.5) \mathrm{a}$ & $10.0(5.3) b$ & 0.002 & 0.488 \\
\hline
\end{tabular}

$418 \mathrm{BW}=$ body weight. $\mathrm{vGRF}=$ vertical ground reaction force. VILR $=$ vertical instantaneous

419 loading rate.

420 Effect sizes are reported as partial $\eta^{2}$, except when Friedman tests were performed (**), where

421 the calculated effect size is reported as average Spearman rho.

$422 *$ geometric means and confidence intervals, back-transformed from $\log _{10}$-transformed data

$423 * *$ values reported are median (IQR)

424 a: different compared to RUN ( $\mathrm{p}<0.05)$

425 b: difference between DU and SS $(\mathrm{p}<0.05)$

426 
427 Table 2. Cycle-averaged mean ground reaction force parameters and joint work at the

428 ankle, knee, and hip during consecutive double unders (DU) and consecutive triple

429 unders (TU) for each participant who successfully completed the TU trial.

\section{Athlete 1}

Athlete 2

Athlete 3

\begin{tabular}{lrrrrrr} 
& \multicolumn{1}{c}{ TU } & \multicolumn{1}{c}{ DU } & \multicolumn{1}{c}{ TU } & \multicolumn{1}{c}{ DU } & \multicolumn{1}{c}{ TU } & \multicolumn{1}{c}{ DU } \\
\hline Contact time (s) & 0.152 & 0.152 & 0.200 & 0.217 & 0.188 & 0.187 \\
vGRF max (BW) & 3.69 & 2.97 & 3.50 & 2.85 & 2.61 & 2.54 \\
$\begin{array}{l}\text { Impulse } \\
\text { (BW·s) }\end{array}$ & 178.2 & 137.2 & 203.8 & 188.5 & 199.6 & 142.3 \\
VILR max (BW/s) & 93.0 & 57.0 & 62.1 & 45.0 & 57.9 & 43.3 \\
& & & & & & \\
\hline Positive work (J) & & & & & & \\
ankle & 47.2 & 36.3 & 67.8 & 56.8 & 62.1 & 45.2 \\
knee & 24.8 & 4.0 & 40.3 & 32.5 & 24.6 & 3.6 \\
hip & 6.9 & 12.6 & 7.0 & 3.6 & 7.6 & 0.6 \\
& & & & & & \\
\hline Negative work (J) & & & & & & \\
ankle & -60.5 & -44.4 & -73.5 & -63.2 & -65.8 & -41.2 \\
knee & -23.5 & -4.0 & -40.9 & -30.6 & -28.2 & -6.1 \\
$\quad$ hip & -1.1 & -2.0 & -7.9 & -4.6 & -11.5 & -2.2
\end{tabular}

$430 \mathrm{BW}=$ body weight. $\mathrm{vGRF}=$ vertical ground reaction force. VILR $=$ vertical

431 instantaneous loading rate.

432

433

434

435

436

437

438

439

440

441

442 
443 Figure 1. Ensemble average curves for vertical ground reaction force and sagittal plane

444 lower limb joint moments during running, double unders, and speed sprint. Shaded

445 areas represent standard deviation.

446

447

$$
\text { Running Double Under }
$$

Speed Step

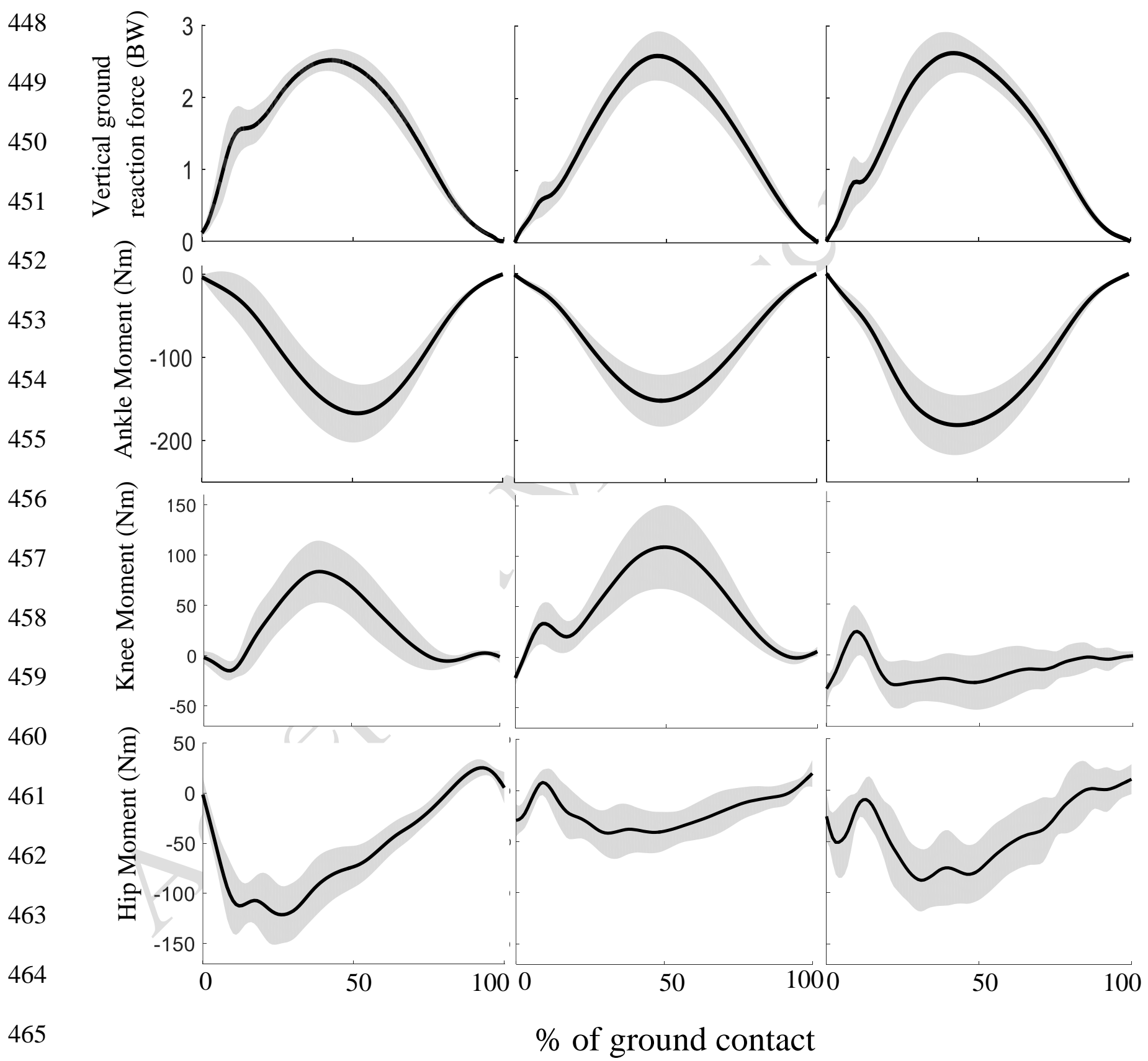

466

$\mathrm{BW}=$ body weight. Ankle: positive is dorsiflexion, negative is plantarflexion. Knee: positive is extension, negative is flexion. Hip: positive is flexion, negative is extension.

467 
469

470

471

472

473

474

475

476

477

478

479

480

481

482

483

484

485

486

487

488

489

490

491

492

493
Figure 2. Ensemble average curves for sagittal plane lower limb joint power during running, double unders, and speed sprint. Shaded areas represent standard deviation. 
494 Figure 3. Joint moments and power during double unders (DU) and triple unders (TU)

495 for each of the three athletes who completed both trials.

496

497

498

499

500

501

502
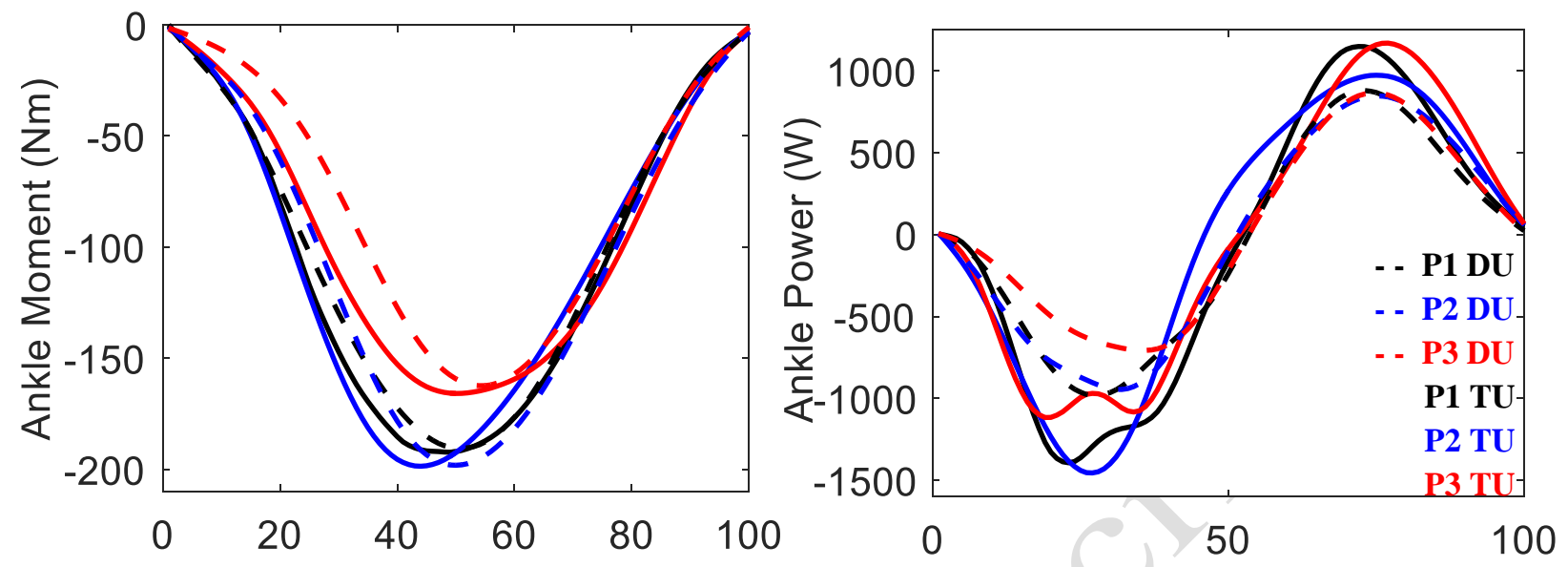

503

504

505

506

507
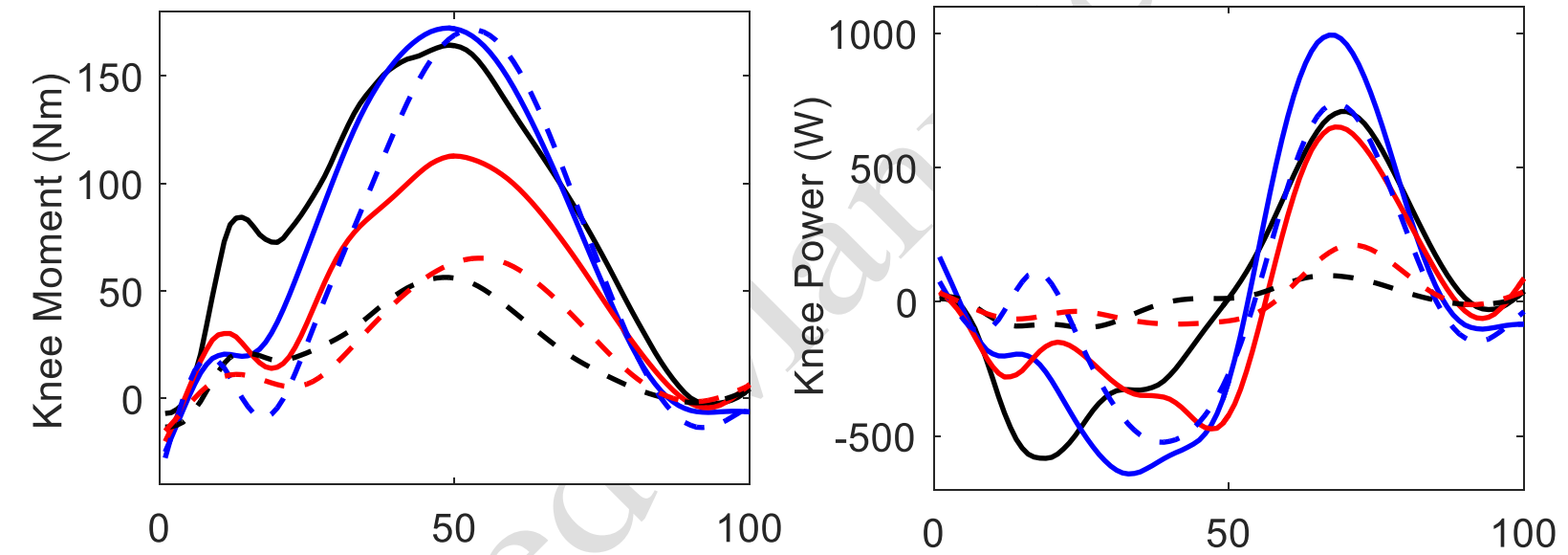

509

510

511

512

513

514 515
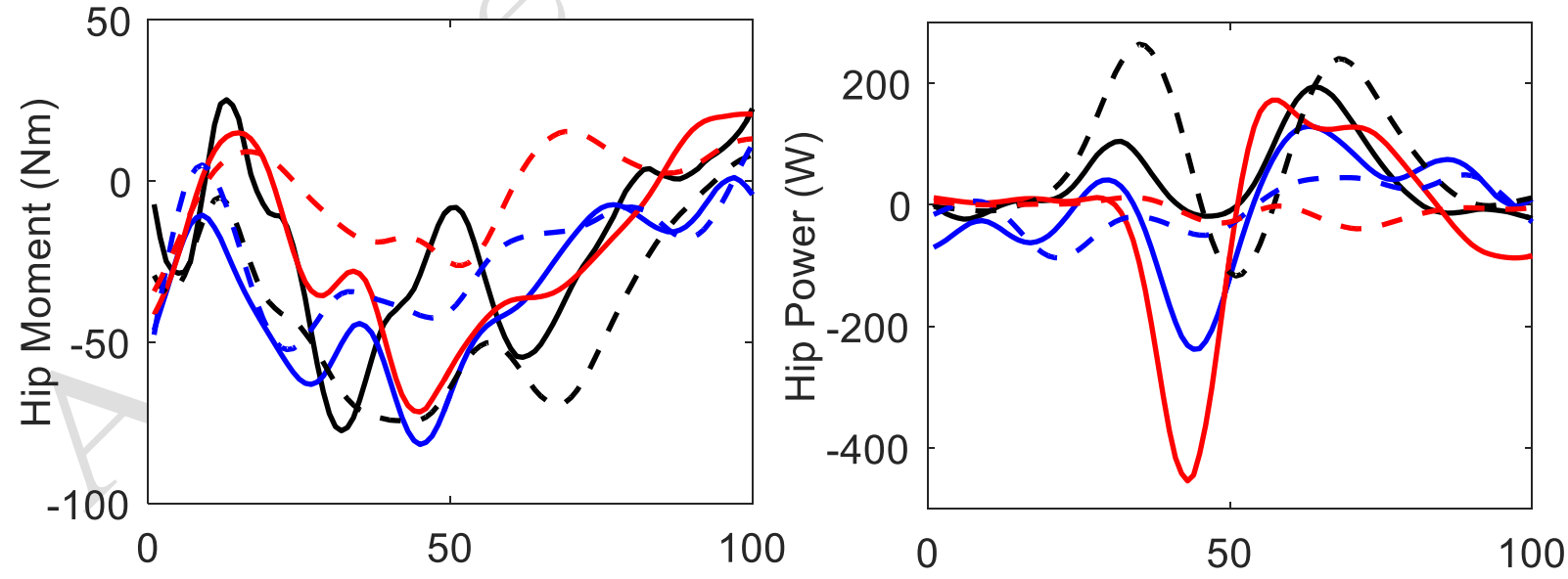

$\%$ of ground contact

Ankle moment: positive is dorsiflexion, negative is plantarflexion. Knee moment: positive is extension, negative is flexion. Hip moment: positive is flexion, negative is extension. 
517 Figure 4: Ensemble average curves for ankle, knee, and hip angular velocity during the

518 ground contact phase of running, double unders, and speed step sprints.

519

520

Runnin

521
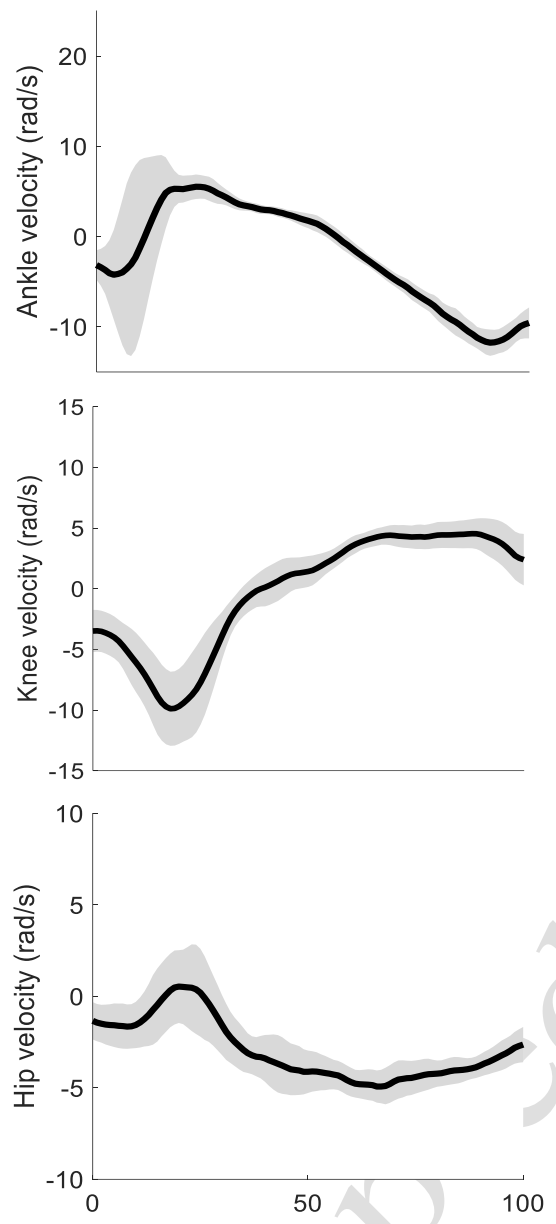

Double
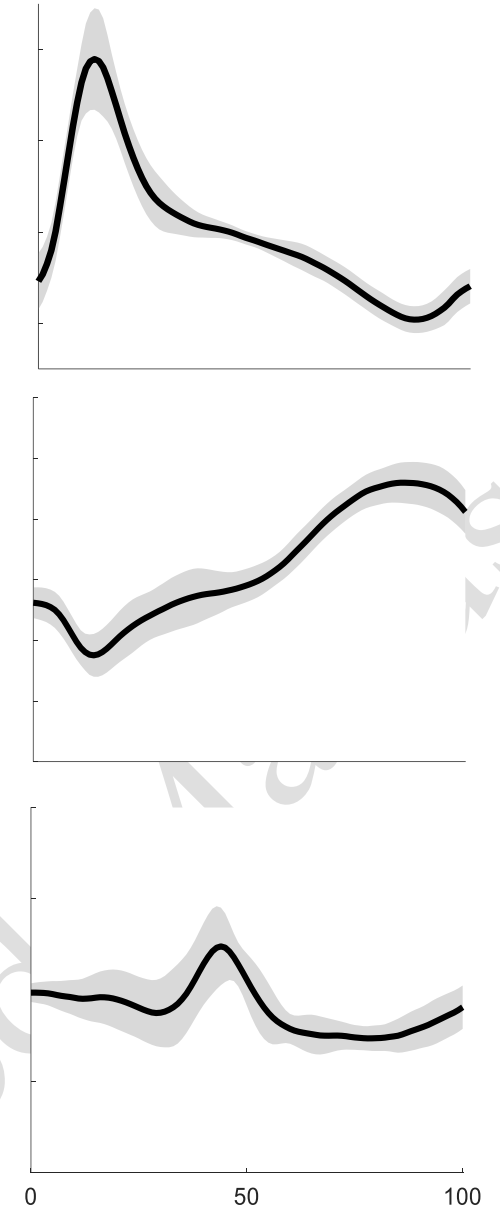

$\%$ of ground contact
Speed
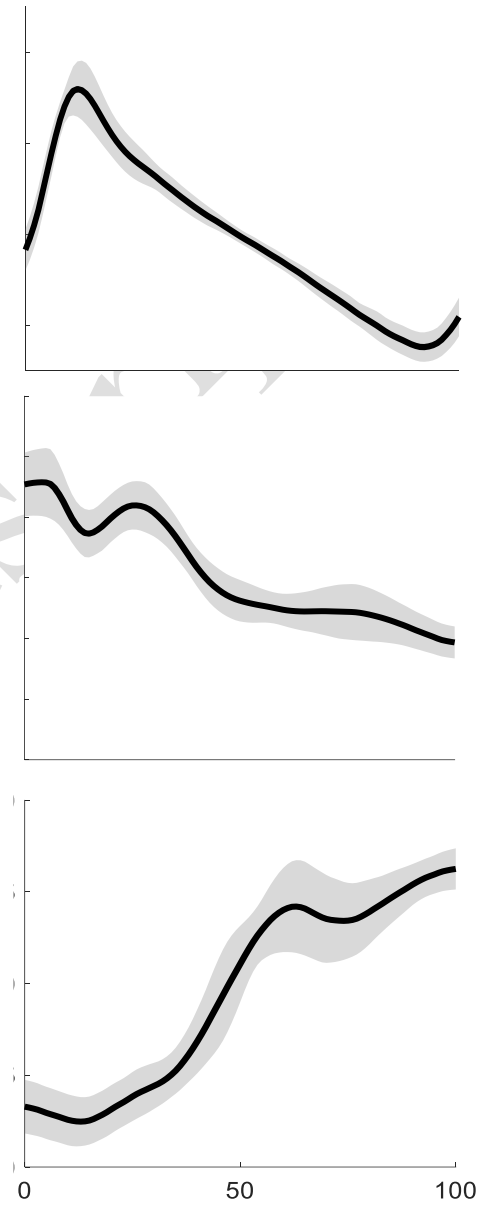

Ankle: positive is dorsiflexion, negative is plantarflexion. Knee: positive is extension, negative is flexion. Hip: positive is flexion, negative is extension. 